3-1993

\title{
Minimizing Channel Density with Movable Terminals
}

\author{
Ronald I. Greenberg \\ Loyola University Chicago, Rgreen@luc.edu
}

Jau-Der Shih

Follow this and additional works at: https://ecommons.luc.edu/cs_facpubs

Part of the Theory and Algorithms Commons, and the VLSI and Circuits, Embedded and Hardware Systems Commons

Author Manuscript

This is a pre-publication author manuscript of the final, published article.

\section{Recommended Citation}

Greenberg, R and S Jau-Der. "Minimizing Channel Density with Movable Terminals." VLSI, 1993. 'Design Automation of High Performance VLSI Systems', Proceedings., Third Great Lakes Symposium on VLSI (GLSV '93).

This Conference Proceeding is brought to you for free and open access by the Faculty Publications and Other Works by Department at Loyola eCommons. It has been accepted for inclusion in Computer Science: Faculty Publications and Other Works by an authorized administrator of Loyola eCommons. For more information, please contactecommons@luc.edu. c) () $\odot$

This work is licensed under a Creative Commons Attribution-Noncommercial-No Derivative Works 3.0 License. (C) IEEE, 1993. 


\title{
Minimizing Channel Density with Movable Terminals
}

\author{
Ronald I. Greenberg and Jau-Der Shih * \\ Electrical Engineering Department \\ University of Maryland \\ College Park, MD 20742
}

\begin{abstract}
We give algorithms to minimize density for channels with terminals that are movable subject to certain constraints. The main cases considered are channels with linear order constraints, channels with linear order constraints and separation constraints, channels with movable modules containing fixed terminals, and channels with movable modules and terminals. In each case, we improve previous results for running time and space by a factor of $L / \lg n$ and $L$, respectively, where $L$ is the channel length, and $n$ is the number of terminals.
\end{abstract}

\section{Introduction}

The channel routing problem has received a great deal of attention in VLSI layout design. Traditionally, channel routers have assumed that the positions of terminals on each side are fixed, and they seek to minimize the width required to route the channel. While determining the width required to route a channel is NP-complete [8], channel density provides a fairly good estimate for channel width. In fact, many existing channel routers achieve widths that are usually within one of the density, e.g., [7].

In this paper we consider the situation in which the orderings of the terminals and components along each side of the channel are fixed, but the exact positions may vary. The existence of movable terminals is quite typical in practice and can be used to reduce the channel density and channel width. When only the ordering of terminals on each side is fixed, Gopal, Coppersmith, and Wong [4] give an $O\left(n^{2}\right)$ algorithm to minimize the channel width ${ }^{1}$, where $n$ is the number of terminals. LaPaugh and Pinter [6] presented an

\footnotetext{
*Supported in part by NSF grant CCR-9109550

${ }^{1}$ This does not contradict the NP-completeness result, due to the use of a model in which there is complete freedom to choose the amount of space between adjacent terminals.
}

$O\left(n^{2} \lg n\right)$ algorithm to minimize the channel density with the additional constraint that the relative positions of the terminals on each side are fixed. That is, the terminals lie on a single top module and a single bottom module, and the only freedom is to shift the modules relative to each other. More recently, Johnson, LaPaugh, and Pinter [5] provided an $O\left(n^{3}\right)$ algorithm to minimize density when there are multiple modules and terminal positions are fixed within each module, but the only other constraint is a fixed order for the modules on each side.

In the above works, however, the resulting channel length may be as large as $p+q$, where $p$ is the number of top terminals and $q$ is the number of bottom terminals (or as large as the sum of the module lengths). In contrast, Cai and Wong $[1,2]$ minimize density for a channel of fixed length $L$ under a wide variety of constraints on the terminal positions. For channels with only linear order constraints (the orderings of the terminals on each side of the channel are fixed), they proposed an $O(p q L)$ algorithm to minimize the channel density. If we add separation constraints (the distance between each pair of consecutive terminals is within a certain range), their running time and space become $O\left(p q L^{3}\right)$ and $O\left(p q L^{2}\right)$, respectively. With multiple modules and fixed terminals within each module, they obtain $O\left(L^{3}\right)$ time and space. If the terminals within the modules are also movable, then the running time and space become $O\left(p q L^{3}\right)$. Cai and Wong describe the practical applicability of these problems and show good reduction of density on sample problems with modest values of $L$.

In this paper we provide more efficient algorithms for these four problems of Cai and Wong $[1,2]$. In each case, we improve the running time by a factor of $L / \lg (p+q)$ and the space by a factor of $L$. (It is easy to also incorporate "position constraints", which specify a set of allowable columns for each terminal, as do Cai and Wong; we will omit further discussion of such constraints in this paper.)

The remainder of this paper is organized as fol- 
lows. In Section 2, we introduce some additional terminology and notation which will be used throughout this paper. Section 3 describes an algorithm to find the minimum channel density for channels with linear order constraints by using a dynamic programming approach. The algorithm is then extended in Sections 4,5 , and 6 to handle channels with separation constraints, channels with movable modules, and channels with movable modules and movable terminals, respectively. Finally, in Section 7 , we provide some concluding remarks.

\section{Preliminaries}

We start this section by giving the problem definition and some notations. We define $t_{1}, t_{2}, \ldots, t_{p}$ and $b_{1}, b_{2}, \ldots, b_{q}$ to be the terminals on the top and bottom side of the channel, which are ordered from left to right. We are given $L$ column positions in which to place the terminals while retaining the given ordering on each side. The goal is to find the positions of the terminals such that the channel density is minimized.

Note that the density at any given column depends only on the fixed order of the terminals on each side and the position of that column within those orderings. Then let $d_{1}(i, j)$ be the density at the column of $t_{i}$ when $t_{i}$ is placed between $b_{j}$ and $b_{j+1}$, let $d_{2}(i, j)$ be the density at the column of $b_{j}$ when $b_{j}$ is placed between $t_{i}$ and $t_{i+1}$, and let $d_{3}(i, j)$ be the density at the column of $t_{i}$ and $b_{j}$ when they are aligned. These density functions can be computed easily in $O(p q)$ time for all possible $i, j$; we assume throughout this paper that these values have been computed and saved. Also define $\delta_{1}^{d}(i, j)$ to be

$$
\delta_{1}^{d}(i, j)=\left\{\begin{array}{ll}
1 & \text { if } d_{1}(i, j) \leq d \\
\infty & \text { if } d_{1}(i, j)>d
\end{array},\right.
$$

and define $\delta_{2}^{d}(i, j)$ and $\delta_{3}^{d}(i, j)$ analogously.

The main idea of our algorithms is as follows. Given a target density $d$, we compute the minimum channel length required to achieve the density. Based on the computed channel length and $L$, we increase or decrease the target density. By using a binary search on all the possible channel densities, we can find the minimum density achievable in length $L$.

\section{Channels with Linear Order Con- straints}

In this section, we give an algorithm to minimize the channel density for channels with linear order con- straints. We begin by showing how to find the minimum channel length at a given target density $d$. To do that, we introduce some subproblems used as the basis for a solution by dynamic programming. (We show in detail only how to find the minimum channel length, but one can readily retrace the computations leading to this result to determine the corresponding terminal placement.)

The length function $L^{d}(i, j)$ is defined to be the minimum number of columns spanned by top terminals $t_{1}, \ldots, t_{i}$ and bottom terminals $b_{1}, \ldots, b_{j}$, with the restriction that each of those columns has density at most $d$ when all the other terminals are placed to the right of both $t_{i}$ and $b_{j}$. If the target density $d$ is unachievable, then $L^{d}(i, j)$ is defined to be $\infty$. We define $L_{1}^{d}(i, j)$ the same way as $L^{d}(i, j)$ but with the constraint that $t_{i}$ is to the right of $b_{j} . L_{2}^{d}(i, j)$ and $L_{3}^{d}(i, j)$ are defined similarly but with the constraint that $t_{i}$ is to the left of $b_{j}$, and $t_{i}$ is aligned with $b_{j}$, respectively. We now show how to compute these functions recursively using the shorthand

$$
L^{d}(i, j)=\min \left\{L_{1}^{d}(i, j), L_{2}^{d}(i, j), L_{3}^{d}(i, j)\right\} .
$$

The final answer to our problem is $L^{d}(p, q)$.

Consider first the computation of $L_{1}^{d}(i, j)$. By the definition of $L_{1}^{d}(i, j), t_{i}$ must be to the right of $b_{j}$. Thus we require one column more than are spanned by $t_{1}, t_{2}, \ldots, t_{i-1}$ and $b_{1}, b_{2}, \ldots, b_{j}$ :

$$
L_{1}^{d}(i, j)=\left(L^{d}(i-1, j)+1\right) \delta_{1}^{d}(i, j) .
$$

Similarly, we can express $L_{2}^{d}(i, j)$ and $L_{3}^{d}(i, j)$ as

$$
L_{2}^{d}(i, j)=\left(L^{d}(i, j-1)+1\right) \delta_{2}^{d}(i, j)
$$

and

$$
L_{3}^{d}(i, j)=\left(L^{d}(i-1, j-1)+1\right) \delta_{3}^{d}(i, j) .
$$

For initial conditions, we have, for $c=1,2,3$,

$$
L_{c}^{d}(0, j)=j \prod_{k=1}^{j} \delta_{c}^{d}(0, k), \quad j=0,1, \ldots, q,
$$

and

$$
L_{c}^{d}(i, 0)=i \prod_{k=1}^{i} \delta_{c}^{d}(k, 0), \quad i=0,1, \ldots, p
$$

where we think of $t_{0}$ and $b_{0}$ as dummy terminals at the left of their respective sides that do not contribute to density. 
Theorem 1 Given a target density d, the minimum channel length subject to linear order constraints can be computed in $O(p q)$ time and space.

Proof. We have already noted that the $\delta$ values can be computed in $O(p q)$ time, and an additional $O(p+q)$ time suffices to determine the initial conditions. Then we compute the values of the three length functions together in order of increasing $i$ and $j$ using the recurrences above. There is a total of $O(p q)$ values to compute, and each can be computed in $O(1)$ time from previously computed values.

Corollary 2 The minimum density of a channel subject to linear order constraints can be found in $O(p q \lg (p+q))$ time and $O(p q)$ space.

Proof. The minimum density problem can be solved by binary search on density, which is at most $p+q$.

\section{Channels with Linear Order Con- straints and Separation Constraints}

In this section, we extend the algorithm of Section 3 to handle channels with linear order constraints and separation constraints. Let the separation constraints have the following form: the distance $s_{i}$ between $t_{i}$ and $t_{i+1}$ must satisfy $l_{i} \leq s_{i} \leq r_{i}$, and the distance $s_{j}^{\prime}$ between $b_{j}$ and $b_{j+1}$ must satisfy $l_{j}^{\prime} \leq s_{j}^{\prime} \leq r_{j}^{\prime}$.

To handle the distance constraints, we have to modify the length functions. Let $L_{1}^{d}(i, j, k)$ and $L_{2}^{d}(i, j, k)$ be defined as in Section 3 but with the restriction that the horizontal distance between $t_{i}$ and $b_{j}$ equals $k$ (in absolute value). We define $L_{3}^{d}(i, j)$ exactly as before. The constraints for the three length functions are illustrated in Figure 1. Then, $L^{d}(i, j)$ is obtained by minimizing over the three types of length functions and all possible $k$ 's.

Consider $L_{1}^{d}(i, j, k)$ first. There are three cases: (1) $t_{i-1}$ is to the right of $b_{j},(2) t_{i-1}$ is to the left of $b_{j}$, and (3) $t_{i-1}$ is aligned with $b_{j}$. And the minimum among the three cases is the minimum channel length. In the first case,

$$
L_{1}^{d}(i, j, k)=\min _{k^{\prime}}\left\{L_{1}^{d}\left(i-1, j, k^{\prime}\right)+k-k^{\prime}\right\} \delta_{1}^{d}(i, j),
$$

with $l_{i-1} \leq k-k^{\prime} \leq r_{i-1}$. Figure $2($ a) illustrates the restriction on $k^{\prime}$. The second case can be analyzed similarly, and we have

$$
L_{1}^{d}(i, j, k)=\min _{k^{\prime}}\left\{L_{2}^{d}\left(i-1, j, k^{\prime}\right)+k\right\} \delta_{1}^{d}(i, j),
$$

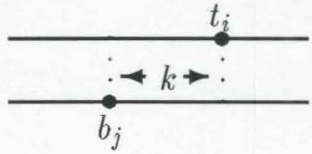

(a)

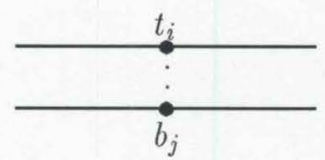

(c)

Figure 1: Three types of length functions: (a) $L_{1}^{d}(i, j, k)(\mathrm{b}) L_{2}^{d}(i, j, k)(\mathrm{c}) L_{3}^{d}(i, j)$

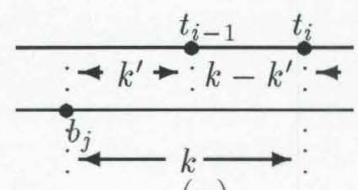

(a)

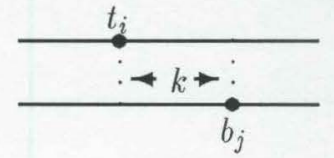

(b)

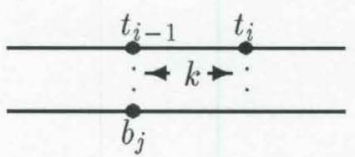

(b)

(c)

Figure 2: Three possibilities of $L_{1}^{d}(i, j, k)$ : (a) $t_{i-1}$ is to the right of $b_{j}$. (b) $t_{i-1}$ is to the left of $b_{j}$. (c) $t_{i-1}$ is aligned with $b_{j}$.

with $l_{i-1} \leq k+k^{\prime} \leq r_{i-1}$. In the third case, which is possible only when $l_{i-1} \leq k \leq r_{i-1}$, we find

$$
L_{1}^{d}(i, j, k)=\left(L_{3}^{d}(i-1, j)+k\right) \delta_{1}^{d}(i, j) .
$$

All three cases are shown in Figure 2. The range of $k$ is $0<k<L$.

From the above argument, $L_{1}^{d}(i, j, k)$ can be expressed as

$$
L_{1}^{d}(i, j, k)= \begin{cases}\left(\min A_{1}\right) \delta_{1}^{d}(i, j) & \text { if } l_{i-1} \leq k \leq r_{i-1} \\ \left(\min A_{2}\right) \delta_{1}^{d}(i, j) & \text { otherwise }\end{cases}
$$

where

$$
A_{1}=\left\{L_{3}^{d}(i-1, j)+k\right\} \cup A_{2},
$$

and

$$
\begin{aligned}
& A_{2}=\left\{\min _{l_{i-1} \leq k-k^{\prime} \leq r_{i-1}}\left\{L_{1}^{d}\left(i-1, j, k^{\prime}\right)+k-k^{\prime}\right\},\right. \\
&\left.\min _{l_{i-1} \leq k+k^{\prime} \leq r_{i-1}}\left\{L_{2}^{d}\left(i-1, j, k^{\prime}\right)+k\right\}\right\} .
\end{aligned}
$$

Similarly, $L_{2}^{d}(i, j, k)$ and $L_{3}^{d}(i, j)$ can be expressed as follows:

$$
L_{2}^{d}(i, j, k)= \begin{cases}\left(\min B_{1}\right) \delta_{2}^{d}(i, j) & \text { if } l_{i-1}^{\prime} \leq k \leq r_{i-1}^{\prime} \\ \left(\min B_{2}\right) \delta_{2}^{d}(i, j) & \text { otherwise }\end{cases}
$$


and

$$
L_{3}^{d}(i, j)=\left\{\begin{array}{c}
\left(\min C_{1}\right) \delta_{3}^{d}(i, j) \\
\text { if }\left[l_{i-1}, r_{i-1}\right] \cap\left[l_{i-1}^{\prime}, r_{i-1}^{\prime}\right] \neq \emptyset \\
\left(\min C_{2}\right) \delta_{3}^{d}(i, j) \text { otherwise }
\end{array}\right.
$$

where

$$
\begin{gathered}
B_{1}=\left\{L_{3}^{d}(i, j-1)+k\right\} \cup B_{2}, \\
B_{2}=\left\{\min _{l_{i-1}^{\prime} \leq k+k^{\prime} \leq r_{i-1}^{\prime}}\left\{L_{1}^{d}\left(i, j-1, k^{\prime}\right)+k\right\},\right. \\
\left.\min _{l_{i-1}^{\prime} \leq k-k^{\prime} \leq r_{i-1}^{\prime}}\left\{L_{2}^{d}\left(i, j-1, k^{\prime}\right)+k-k^{\prime}\right\}\right\}, \\
C_{1}=\left\{L_{3}^{d}(i-1, j-1)+\max \left\{l_{i-1}, l_{i-1}^{\prime}\right\}\right\} \cup C_{2}, \\
C_{2}=\left\{\min _{\left(m, k^{\prime}\right) \in I_{m, k^{\prime}}}\left\{L_{1}^{d}\left(i-1, j-1, k^{\prime}\right)+m\right\},\right. \\
\left.\min _{\left(m, k^{\prime}\right) \in J_{m, k^{\prime}}}\left\{L_{2}^{d}\left(i-1, j-1, k^{\prime}\right)+m\right\}\right\}, \\
I_{m, k^{\prime}}=\left\{\left(m, k^{\prime}\right) \mid l_{i-1} \leq m \leq r_{i-1}\right. \text { and } \\
\left.l_{i-1}^{\prime} \leq m+k^{\prime} \leq r_{i-1}^{\prime}\right\},
\end{gathered}
$$

and

$$
\begin{aligned}
J_{m, k^{\prime}}=\left\{\left(m, k^{\prime}\right) \mid l_{i-1}^{\prime}\right. & \leq m \leq r_{i-1}^{\prime} \text { and } \\
l_{i-1} & \left.\leq m+k^{\prime} \leq r_{i-1}\right\} .
\end{aligned}
$$

Theorem 3 Given a target density $d$, the minimum channel length subject to linear order constraints and separation constraints can be computed in $O\left(p q L^{2}\right)$ time and $O(p q L)$ space.

Proof. We compute values of the length functions in order of increasing $i, j$ and $k$, and then the minimum channel length is

$$
\min \left\{\min _{0<k<L} L_{1}^{d}(p, q, k), \min _{0<k<L} L_{2}^{d}(p, q, k), L_{3}^{d}(p, q)\right\} .
$$

There are $O(p q L)$ values of $L_{1}^{d}$ and $L_{2}^{d}$ to be computed, and each can be computed from previously computed values in $O(L)$ time. In addition, there are $O(p q)$ values of $L_{3}^{d}$ to be computed, each in time $O\left(L^{2}\right)$.

Corollary 4 The minimum density of a channel subject to linear order constraints and separation constraints can be found in $O\left(p q L^{2} \lg (p+q)\right)$ time and $O(p q L)$ space.

\section{Channels with Movable Modules}

This section considers the problem of channels with movable modules, but the terminals inside the modules are fixed. We first augment the set of terminals to include the endpoints of the modules. Then we insert pseudo-terminals on the modules until every column in the modules contains a terminal or a pseudo-terminal as in [2]. As a result, the separation constraints between terminals inside a top module have the form $l_{i}=r_{i}=1$ (an adjacency constraint), and the separation constraints between the right endpoint of a top module and the left endpoint of the module immediately to its right are $l_{i}=1$, and $r_{i}=\infty$. (The constraints on the bottom are similar.) Now we can see this problem as a channel subject to linear order constraints and special separation constraints.

The length functions used in this section are as defined in Section 3. The approach to calculate these length functions is the same except for a modification to handle adjacency constraints. Using the notational shorthand

$$
L_{x, y}^{d}(i, j)=\min \left\{L_{x}^{d}(i, j), L_{y}^{d}(i, j)\right\},
$$

we have:

$$
\begin{aligned}
& L_{1}^{d}(i, j)= \begin{cases}\left(L^{d}(i-1, j)+1\right) \delta_{1}^{d}(i, j) & \text { if } r_{i-1}=\infty \\
\left(L_{1,3}^{d}(i-1, j)+1\right) \delta_{1}^{d}(i, j) & \text { if } r_{i-1}=1\end{cases} \\
& L_{2}^{d}(i, j)= \begin{cases}\left(L^{d}(i, j-1)+1\right) \delta_{2}^{d}(i, j) & \text { if } r_{j-1}^{\prime}=\infty \\
\left(L_{2,3}^{d}(i, j-1)+1\right) \delta_{2}^{d}(i, j) & \text { if } r_{j-1}^{\prime}=1\end{cases} \\
& L_{3}^{d}(i, j)=\left\{\begin{array}{c}
\left(L^{d}(i-1, j-1)+1\right) \delta_{3}^{d}(i, j) \\
\text { if } r_{i-1}=r_{j-1}^{\prime}=\infty \\
\left(L_{1,3}^{d}(i-1, j-1)+1\right) \delta_{3}^{d}(i, j) \\
\text { if } r_{i-1}=1 \text { and } r_{j-1}^{\prime}=\infty \\
\left(L_{2,3}^{d}(i-1, j-1)+1\right) \delta_{3}^{d}(i, j) \\
\text { if } r_{i-1}=\infty \text { and } r_{j-1}^{\prime}=1 \\
\left(L_{3}^{d}(i-1, j-1)+1\right) \delta_{3}^{d}(i, j) \\
\text { if } r_{i-1}=r_{j-1}^{\prime}=1
\end{array}\right.
\end{aligned}
$$

and

$$
L^{d}(i, j)=\min \left\{L_{1}^{d}(i, j), L_{2}^{d}(i, j), L_{3}^{d}(i, j)\right\} .
$$

Theorem 5 Given a target density d, the minimum channel length for channels with movable modules can be computed in $O\left(L^{2}\right)$ time and space.

Proof. We can compute $L_{1}^{d}(i, j), L_{2}^{d}(i, j)$, and $L_{3}^{d}(i, j)$ from previously computed values in $O(1)$ time. Including the pseudo-terminals, there are $O(L)$ terminals on each side of the channel, which yields $O\left(L^{2}\right)$ length function values to be computed.

Corollary 6 The minimum density of a channel with movable modules can be solved in $O\left(L^{2} \lg (p+q)\right)$ time and $O\left(L^{2}\right)$ space. 


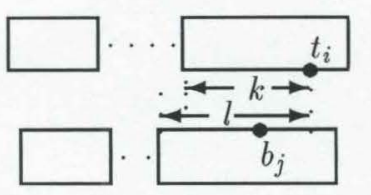

(a)

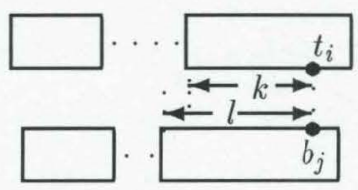

(c)

Figure 3: Three types of length functions: $L_{1}^{d}(i, j, k, l)$ (b) $L_{2}^{d}(i, j, k, l)$ (c) $L_{3}^{d}(i, j, k, l)$

\section{Channels with Movable Terminals and Modules}

In this section, we consider channels with movable terminals and modules. That is, the modules on each side of the channel are movable as in Section 5, but we also allow the terminals within the modules to be movable. To handle this situation, we have to introduce new definitions and length functions.

Define a left terminal to be the leftmost terminal of a module, a left endpoint to be the left endpoint of a module, and a right endpoint to be the right endpoint of a module. Now augment the set of terminals to include the endpoints of the modules. The length functions have four variables $i, j, k$, and $l$ as illustrated in Figure 3 ; here $k$ and $l$ represent the distance from the rightmost of $t_{i}$ and $b_{j}$ to the left edges of their modules. The length function $L^{d}(i, j)$ is equal to the minimum of the three types of length functions for all possible $k$ 's and l's (where each length function accounts for the lengths of the modules containing $t_{1}, t_{2}, \ldots, t_{i}$ and $b_{1}, b_{2}, \ldots, b_{j}$ ).

In order to compute the length functions, we classify the terminals into four types: left endpoints, right endpoints, left terminals, and others. With a lengthy case analysis based on the types of $t_{i}$ and $b_{j}$, we can minimize density in $O\left(p q L^{2} \lg (p+q)\right)$ time and $O\left(p q L^{2}\right)$ space.

\section{Conclusion}

We have presented algorithms to minimize the channel density for a variety of problems. These algorithms improve the previous known results by $O(L / \lg (p+q))$ in running time and $O(L)$ in space. These algorithms can also easily be extended to chan- nels with exits or channels with irregular boundaries as in [1] without increasing the complexity. In the process of minimizing density for a fixed channel length, we have provided even more efficient algorithms to minimize length at a fixed density. By running the latter type of algorithm $O(p+q)$ times, we can also minimize more complex cost measures, such as area (where density is treated as width) in a channel of length at most $L$.

For the case of movable modules with fixed terminals, density can be minimized in a channel of length $L$ in $O\left(n^{3} \lg n\right)$ time independent of $L$ (which is an improvement for large $L$ ) using the method of Chao and LaPaugh [3]. However, their method can not be extended to handle channels with movable terminals. An interesting open question is to solve other variations of the problem in time polynominal in $n$ only.

\section{References}

[1] Y. Cai and D. F. Wong. Minimizing channel density by shifting blocks and terminals. In IEEE International Conference on Computer-Aided Design (ICCAD-91), pages 524-527. IEEE Computer Society Press, 1991.

[2] Y. Cai and D. F. Wong. Optimal channel pin assignment. IEEE Trans. Computer-Aided Design of Integrated Circuits, 10(11):1413-1424, Nov. 1991.

[3] L.-F. Chao and A. S. LaPaugh. Finding all minimal shapes in a routing channel. Technical Report YALEU/DCS/TR-384, Yale University Department of Computer Science, Aug. 1992.

[4] I. S. Gopal, D. Coppersmith, and C. Wong. Optimal wiring of movable terminals. IEEE Trans. Computers, C-32(9):845-858, Sept. 1983.

[5] D. S. Johnson, A. S. LaPaugh, and R. Y. Pinter. Minimizing channel density by lateral shifting of components. Manuscript, 1992.

[6] A. S. LaPaugh and R. Y. Pinter. On minimizing channel density by lateral shifting. In IEEE International Conference on Computer-Aided Design (ICCAD-83), pages 121-122. IEEE Computer Society Press, 1983.

[7] J. Reed, A. Sangiovanni-Vincentelli, and M. Santomauro. A new symbolic channel router: YACR2. IEEE Trans. Computer-Aided Design of Integrated Circuits, CAD-4(3):208-219, July 1985.

[8] T. G. Szymanski. Dogleg channel routing is NPcomplete. IEEE Trans. Computer-Aided Design of Integrated Circuits, CAD-4(1):31-41, Jan. 1985. 